\title{
ALGO+ Uma ferramenta para o apoio ao ensino de Algoritmos e Programação para alunos iniciantes
}

\author{
Érico Amaral ${ }^{1}$, Alex Camargo ${ }^{1}$, Marina Gomes ${ }^{1}$, César Richa ${ }^{2}$, Liliane Becker ${ }^{1}$ \\ ${ }^{1}$ Universidade Federal do Pampa (UNIPAMPA) \\ Bagé - RS - Brasil \\ ${ }^{2}$ Universidade Federal de Santa Catarina (UFSC) \\ Santa Catarina - RS - Brasil \\ \{ericohoffamaral, alexcamargoweb, marina93, huegelcesar, \\ lilianebb\} @gmail.com
}

\begin{abstract}
This article presents the project, construction, and validation of a tool to support the process of teaching and learning logic and programming for beginners. The conception of the research is fundamentally based on concepts and approaches of learning applied in a Web Portal, through which the student can realize a construction of the knowledge about the programming, autonomously and at a distance. As results of the initial experiments, it was possible to recognize a satisfactory performance of the students who used the learning environment. Finally, the analysis of a research instrument pointed to the effectiveness in the adoption of the Something + for the purpose that was implemented..
\end{abstract}

Resumo. Este artigo apresenta o projeto, construção e validação de uma ferramenta para o apoio ao processo de ensino e de aprendizagem de lógica e programação, para alunos iniciantes. A concepção desta pesquisa é fundamentalmente baseada em conceitos e teorias de aprendizagem aplicadas em um Portal Web, por meio do qual o estudante pode realizar a construção do conhecimento sobre programação, de forma autônoma e à distância. Como resultados dos experimentos iniciais, foi possivel reconhecer um desempenho satisfatório dos alunos que utilizaram o ambiente de aprendizagem. Finalmente, a análise de um instrumento de pesquisa apontou para a efetividade na adoção do Algo+ para a finalidade que foi implementado.

\section{Introdução}

Os conteúdos abordados na disciplina Algoritmos e Programação são considerados de extrema importância para o êxito na formação de alunos que ingressam em cursos na área de tecnologia, nos diversos níveis do ensino como: técnicos, tecnológicos e bacharelados. Os conhecimentos adquiridos nesta disciplina vão da lógica de programação ao entendimento de conceitos e da abstração necessária para a resolução de problemas, conhecimentos necessários para a formação de alunos da área de computação e afins.

O objetivo principal abordado no estudo de algoritmos é reconhecer os conceitos básicos para o seu desenvolvimento, fornecendo ao aluno uma visão crítica e 
sistemática, necessária para o desenvolvimento de soluções algorítmicas. Contudo, é reconhecido que os estudante apresentam, nestas disciplinas, um alto nível de dificuldade na compreensão dos conteúdos (SILVA et al. 2009), o que é comprovado estatisticamente, visto que as turmas iniciam em média com 50 matriculados e, em poucos meses, a quantidade de alunos regulares cai para aproximadamente 20, constatando-se uma taxa entre $50 \%$ e $60 \%$ de reprovação ou desistência (ROCHA, 1993; DETERS, 2008). Tais problemas de aprendizagem podem ser apontados por diferentes aspectos como: uma base matemática insuficiente, limitações de ensino do professor, materiais de apoio não efetivos ou inexistentes, dificuldade de entendimento e abstração dos alunos, entre outros.

O desenvolvimento desta pesquisa teve como objetivo levantar requisitos, analisar, projetar e implementar uma solução para apoio ao processo de ensino/aprendizagem da disciplina de Algoritmos e Programação, a qual foi denominada Algo+. A proposta deste Portal se diferencia dos demais objetos de aprendizagem, pois está fundamentalmente calcada sobre conceitos e métodos de aprendizagem como o Ciclo de Aprendizagem Experimental de Kolb (KOLB, 2014), respeitando as quatro etapas básicas para o aprendizado efetivo: sentir, observar, pensar e o fazer. Este projeto foi desenvolvido a partir de um plano de ensino adaptado, contemplando diferentes estilos cognitivos, aliado a uma estrutura objetiva e clara, visando desta forma proporcionar, ao aluno iniciante, um ambiente atrativo, motivador e de fácil utilização.

A fim de demonstrar este estudo, a presente pesquisa está estruturada da seguinte forma: na seção 2 é apresentada a base teórica, a qual serviu de norte para o trabalho; a seção 3 descreve os métodos e técnicas adotadas; a quarta seção descreve a implementação e discussão dos resultados alcançados e; por fim a seção 5 aponta as conclusões obtidas.

\section{Referencial Teórico}

\subsection{Ensino Tradicional da Lógica de Programação}

O ensino de programação é realizado, tradicionalmente, por meio da exposição do conteúdo pelo professor, aquele que detém o conhecimento e o repassa, baseado na apresentação de teoria e exemplos básicos e proposição de exercícios. Porém, muitas vezes esse método não se adequa as necessidades dos alunos, pois não considera as diferenças entre alunos, suas aptidões e dificuldades, principalmente em conteúdos para os quais habilidades e raciocínio lógico são extremamente necessários (GOMES, 2008).

Um dos problemas do modo tradicional de ensino em algoritmos é a dificuldade de motivar os alunos, de fazer com que se interessem pela disciplina e fazê-los entender que seu conteúdo é importante para sua formação (BORGES, 2000). Outra dificuldade é a de que nem sempre é possível o professor disponibilizar um acompanhamento individualizado, devido ao grande número de alunos em sala de aula, baixa disponibilidade de tempo e a necessidade de cumprimento de um plano de trabalho.

Neste sentido, o ensino tradicional evidencia duas situações: estudantes habituados a serem indivíduos passivos dentro do ambiente escolar e estudantes/professores limitados pelo tempo. Segundo Wang (2015), é necessário emancipar o aluno, de forma que o mesmo possa "aprender a aprender" e validar seus próprios conhecimentos. Assim, a busca por novas metodologias e ferramentas 
VI Congresso Brasileiro de Informática na Educação (CBIE 2017)

Anais do XXVIII Simpósio Brasileiro de Informática na Educação (SBIE 2017)

computacionais de ensino, que estimulem o aprendizado de programação e auxiliem os alunos no desenvolvimento de suas habilidades cognitivas, sua forma de raciocinar e solucionar problemas são necessárias.

\subsection{Trabalhos Correlatos}

A construção de ambientes que estimulem e apresentem uma interface amigável para colaborar na formação dos conceitos sobre programação, através do uso de uma linguagem de programação visual, tem obtido uma notoriedade significativa (Fincher et al. 2010; Resnick et al. 2009). Nos últimos anos várias pesquisas propuseram abordagens para apoiar o processo de ensino-aprendizagem de programação, que têm sido marcadas pelo desenvolvimento de novos recursos.

Dentre os recursos voltados para desenvolver bons hábitos em programação estão o software colaborativo HabiPro e o ambiente Leco-EAD. O HabiPro tem como objetivo não ensinar programação e sim estimular os alunos iniciantes a adquirirem habilidades como entendimento e reflexão da estrutura do algoritmo (VIZCAINO, 2000). O ambiente LeCo-EAD utiliza um sistema companheiro de aprendizagem para ensino a distância na internet, ele é composto por múltiplos agentes companheiros de ensino com comportamentos próprios e sempre disponíveis para interagir com os estudantes, contemplando diferentes táticas de ensino (FARACO,2004).

\section{Metodologia}

Vislumbrando a construção e disponibilização de uma solução de apoio ao processo de ensino e de aprendizagem em Algoritmos e Programação, está pesquisa descreve a implementação e aplicação de um instrumento, na forma de Portal Web, projetado e desenvolvido com base em fundamentadas teorias de aprendizagem. Esta proposta disponibiliza ao professor uma ferramenta que poderá ser adotada, de forma complementar, nas disciplinas iniciais de programação, ou ser utilizada por estudantes, de forma individualizada, para a aprendizagem ou reforço da lógica e programação.

A fim de alcançar os objetivos traçados neste estudo, adotou-se um conjunto de procedimentos sistemáticos e intensivos para a obtenção de informações e análise dos resultados, o que segundo Lehfeld (1991) caracteriza-se como uma metodologia científica. Quanto aos objetivos, este trabalho pode ser classificado como uma pesquisa exploratória, visto que define critérios e métodos no intuito de obter informações relevantes sobre o objeto de pesquisa (BERVIAN et al. 2006). No intuito de entender o desenvolvimento desta pesquisa elaborou-se um digrama com a apresentação do conjunto de ações realizadas, organizadas em 5 etapas previamente estruturadas, conforme ilustra a Figura 01.

\begin{tabular}{|c|c|c|c|c|}
\hline $\begin{array}{c}\text { Definição } \\
\text { Problema de } \\
\text { Pesquisa }\end{array}$ & $\begin{array}{c}\text { Estudo e } \\
\text { definição } \\
\text { Referencial } \\
\text { teórico }\end{array}$ & $\begin{array}{c}\text { Proposta e } \\
\text { Estudo sobre } \\
\text { tecnologias }\end{array}$ & $\begin{array}{c}\text { Projeto, } \\
\text { Implementação } \\
\text { e Testes Portal }\end{array}$ & $\begin{array}{c}\text { Análise de } \\
\text { resultados e } \\
\text { Conclusões }\end{array}$ \\
\hline $\mathbf{1}$ & $\mathbf{2}$ & $\mathbf{3}$ & $\mathbf{4}$ & $\mathbf{5}$ \\
\hline
\end{tabular}

Figura 01. Estrutura de desenvolvimento da pesquisa

O planejamento das atividades partiu da definição do problema de pesquisa na etapa 1, a etapa 2 contemplou estudo e levantamento do referencial teórico para servir 
de base a construção da proposta (Portal), a definição do Portal Algo+, tecnologias adotadas, o projeto, implementação e testes do software foram realizados nas etapas $3 \mathrm{e}$ 4 respectivamente. Por fim a avaliação dos resultados, com base nos experimento de utilização do Portal, uma análise de desempenho dos alunos no ambiente e conclusões foi realizada na quinta etapa.

\section{Algo+ Uma Proposta de Ferramenta para o Apoio ao Ensino de Algoritmos e Programação}

\subsection{A estrutura do Algo+}

Dentre as atuais tecnologias de apoio ao ensino, a Internet destaca-se como sendo o principal meio para o intercâmbio de informações, além dos limites da sala de aula convencional. Com isso, novas propostas podem servir como elemento inovador, diferenciador e dinamizador das aulas, motivando professores e alunos, desenvolvendo a criatividade mútua, assim como, a capacidade de pesquisa e interação social. A ideia principal de explorar o potencial das recentes tecnologias computacionais, para serem somadas à elaboração de atividades em sala de aula, resultou na implementação do Portal Algo+, criado para possibilitar que o acesso aos conteúdos referentes ao ensino de lógica computacional e linguagens de programação possam ser abordados, de forma simples, pelos professores e alunos. A Figura 02 mostra a arquitetura projetada e desenvolvida para o Portal.

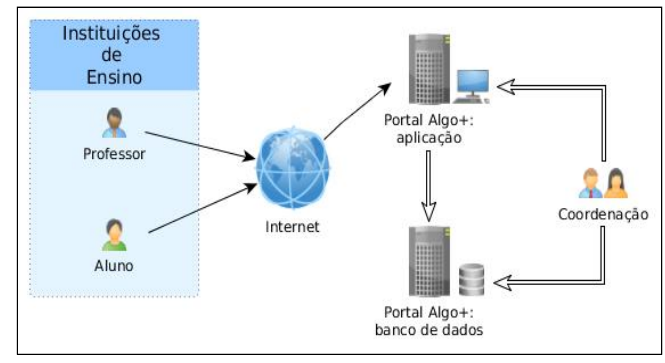

Figura 02. Arquitetura do Algo+

\section{Tabela 01. Módulos do Algo+}

\begin{tabular}{|c|c|}
\hline Módulo & Conteúdo \\
\hline 01 & Lógica de Programação \\
\hline 02 & $\begin{array}{l}\text { Introdução a Programação, variáveis e } \\
\text { constantes }\end{array}$ \\
\hline 03 & $\begin{array}{l}\text { Linguagem Scratch (variáveis, estrutura } \\
\text { condicional e de repetição, listas) }\end{array}$ \\
\hline 04 & $\begin{array}{l}\text { App Inventor (variáveis, estrutura } \\
\text { condicional e de repetição, listas, BD, API } \\
\text { Google) }\end{array}$ \\
\hline 05 & Linguagem C - Estrutura Condicional \\
\hline 06 & Linguagem C - Estrutura de Repetição \\
\hline 07 & $\begin{array}{l}\text { Linguagem C- Manipulação de Strings, } \\
\text { Vetores e Matrizes }\end{array}$ \\
\hline 08 & Linguagem C - Funções e Procedimentos \\
\hline
\end{tabular}

O Algo+ possui duas interfaces, uma de coordenação e outra de uso comum, entre aluno e professor. Em ambos os casos, as informações ficam centralizadas no banco de dados próprio. Para o uso do Portal, inicialmente, o professor envia um e-mail para a coordenação solicitando o acesso à ferramenta. Posteriormente, com o acesso liberado, o professor pode criar turmas e colaborar com o repositório de questões. Após essas etapas, o aluno se inscreve em alguma das turmas disponíveis e a matrícula é validada pelo professor responsável. Finalmente, é disponibilizado ao aluno o conteúdo e as avaliações de cada módulo. A ferramenta obtém o resultado da avaliação e o disponibiliza para visualização pelo professor e pelo aluno, nesse caso, na forma de um certificado.

O Portal é constituído sobre um sistema de cadastro que permite o registro das ações dos alunos a fim de que o professor possa acompanhar o seu desempenho e evolução no ambiente. O professor, igualmente, poderá cadastrar turmas nas quais os alunos serão vinculados a um dos dois perfis disponíveis: aqueles que estudarão apenas uma linguagem de programação tradicional; e os que utilizarão diferentes paradigmas 
VI Congresso Brasileiro de Informática na Educação (CBIE 2017)

Anais do XXVIII Simpósio Brasileiro de Informática na Educação (SBIE 2017)

durante a disciplina. De acordo com este perfil, os conteúdos do portal são dinamicamente selecionados e exibidos para o aluno, conforme seu avanço pelos módulos do curso referente à sua turma.

Com o intuito de tornar o Algo+ uma ferramenta efetiva, a organização dos conteúdos disponibilizados ao aluno em cada módulo, segue uma adaptação da proposta de Amaral (2015), com um conjunto de conteúdos fundamentalmente avaliados e sincronizados de forma a estimular e permitir ao estudante a construção do conhecimento sobre lógica e programação, adotando paradigmas de programação distintos como programação com blocos visuais (Scratch), desenvolvimento para dispositivos móveis (AppInventor) e um paradigma estruturado tradicional (Linguagem C). A sequência de assuntos por módulo pode ser observada na Tabela 01 .

A fim de permitir a autonomia do aluno no desenvolvimento de seus estudos, o Portal disponibiliza um conjunto de recursos midiáticos, que objetiva atender os diferentes estilos de aprendizagem, com 6 abas (Figura 03) a seguir: Introdução e contextualização do Módulo; Downloads (materiais de apoio); Material Teórico (conteúdo específico para leitura); Multimídia (um conjunto de vídeos/áudios relacionados ao Módulo atual); Atividades Formativas (exercícios com feedback automático, seguindo Saikkonen et al. (2001) e; Atividade Avaliativa (10 questões). Cabe ressaltar que as questões que compõem a avaliação são cadastradas pelos professores no sistema e, aleatoriamente selecionadas de acordo com o módulo corrente, visando assim, que o aluno realize uma prova única, distinta dos demais.

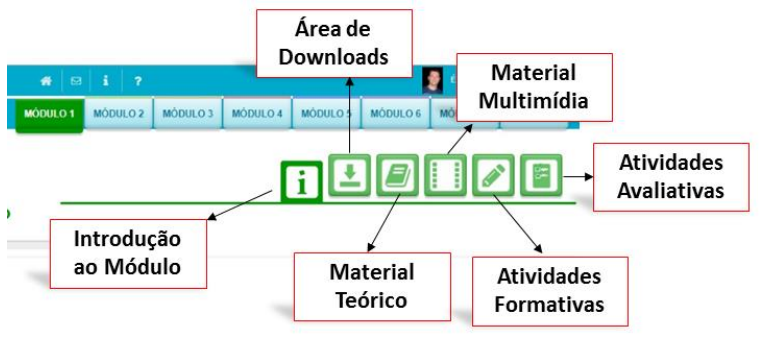

Figura 03. Conteúdos dos módulos

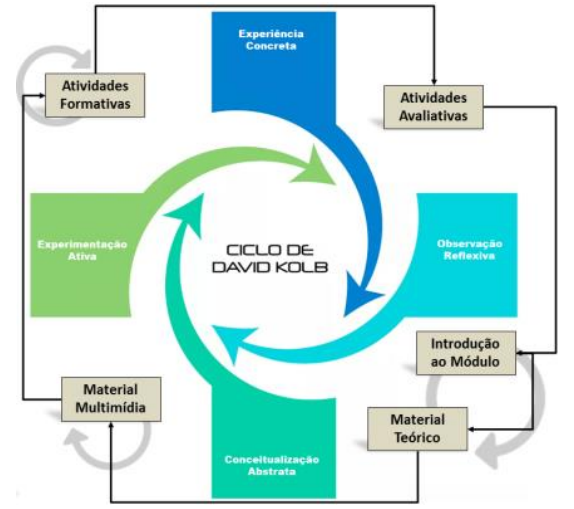

Figura 04. Conteúdos Algo+ alinhados ao Ciclo de Aprendizagem de Kolb

Em relação aos materiais de cada módulo, buscou-se nesta proposta uma composição teoricamente embasada, buscando uma interação positiva e satisfatória, do aprendiz com o ambiente. Desta forma, a concepção dos conteúdos encontra-se de forma a permitir um ciclo, onde o estudante poderá a cada interação retornar e avançar nas suas atividades. Agregou-se ainda, na construção dos módulos, características da Aprendizagem Significativa (AUSUBEL, 2003) que permitam o discente recuperar informações já estudadas em módulos anteriores, além da disponibilização de materiais com diferentes mídias, respeitando a regra de multimodalidade de Do Canto et. al, (2013). Por fim, na Figura 04 é demonstrada a base para o planejamento de todos os módulos, a qual segue o Ciclo de Aprendizagem Experiencial (KOLB, 1984) com as atividades permeadas entre a observação reflexiva, conceitualização abstrata, experimentação ativa e experiência concreta. 
VI Congresso Brasileiro de Informática na Educação (CBIE 2017)

Anais do XXVIII Simpósio Brasileiro de Informática na Educação (SBIE 2017)

\subsection{Tecnologias e Recursos Adotados}

A área de ensino e aprendizagem de linguagens de programação vem se adaptando de forma natural às novas tecnologias por meio da utilização de práticas que exploram de diferentes formas as soluções disponíveis. Para o uso efetivo dos elementos computacionais abordados na literatura é necessário um estudo e desenvolvimento de práticas pedagógicas que integrem os conceitos básicos de programação aos novos padrões de sistemas e recursos.

O conjunto de atividades metodologicamente planejadas neste trabalho, conforme descrito na seção anterior, permitiram a execução das ações de pesquisa deste estudo, possibilitando, desse modo, construir uma solução fundamentalmente alinhada a teorias de aprendizagem com o suporte de abordagens computacionais. $\mathrm{O}$ funcionamento do Portal Algo+ foi concebido para ter a sua execução via $W e b$, através de um navegador web. Com isso, não é necessária nenhuma instalação por parte do usuário, bastando apenas indicar o seu endereço disponível na rede (URL). As principais tecnologias utilizadas foram: HTML (HyperText Markup Language), jQuery, CSS (Cascading Style Sheet), Hot Potatoes, MySQL e PHP (HiperText Preprocessor).

O HTML, versão 5, foi utilizado por ser uma linguagem de marcação predominantemente usada para descrever conteúdo ou dados na Web. Juntamente às CSSs, versão 3, foi possível definir estilos padrão para os elementos que compõem a interface do Portal. Em conjunto com as linguagens HTML e CSS, também foi utilizado o JavaScript (framework jQuery), uma linguagem de programação client-side, ou seja, possibilita que os scripts sejam processados pelo computador do usuário, sem a necessidade de passar pelo servidor de aplicação. O seu uso pode ser exemplificado na validação de formulários, exibição de menus e direcionamento das páginas (navegação guiada).

De outra forma, como principal linguagem server-side (processada no servidor) foi adotado o PHP, uma linguagem de uso geral direcionada para o desenvolvimento de aplicações inseríveis dentro da estrutura do HTML. O PHP é responsável, predominantemente, por processar os dados a serem apresentados no $\mathrm{Algo}^{+}$, como as notas e informações dos alunos armazenadas no banco de dados. Em relação à base de dados adotada, optou-se por utilizar o Sistema Gerenciador de Banco de Dados (SGBD) MySQL pelo fato de apresentar características open-source bem como uma ampla documentação e discussão mantida pela comunidade de desenvolvedores. Por fim, diferentes atividades do tipo "pergunta-resposta" foram incluídas através do software educacional Hot Potatoes (ROZAS, 2008), sob a forma digital de publicação na Web no formato HTML. Dessa maneira, a ferramenta interage dinamicamente com o usuário enquanto as questões são preenchidas, igualmente, ao final exibe a resolução de cada exercício proposto.

\subsection{Experimentos, Resultados e Discussões}

A fim de verificar a usabilidade e efetividade do Portal Algo+, no processo de ensinoaprendizagem de algoritmos e programação, dois professores da disciplina Algoritmos foram convidados a utilizar este recurso como instrumento de apoio em suas atividades didáticas. Foram liberados, para estes docentes, os dois primeiros módulos do Algo+, os 
VI Congresso Brasileiro de Informática na Educação (CBIE 2017)

Anais do XXVIII Simpósio Brasileiro de Informática na Educação (SBIE 2017)

quais contemplam os conceitos de lógica de programação, construção de algoritmos, uso de variáveis (tipos) e formas de representação de algoritmos.

O experimento realizado aconteceu com duas turmas da disciplina de Algoritmos e Programação, a turma A, composta por 31 alunos do curso de Engenharia de Computação e, a turma B, composta por 16 alunos dos cursos de Engenharia de Produção, Engenharia de Alimentos, Engenharia de Energias e Física, totalizando 47 alunos. Estes discentes acessaram o ambiente por duas semanas, tendo o prazo de sete dias para a realização/estudo de todas as atividades de cada módulo. Ao utilizarem o portal os alunos tiveram acesso aos materiais introdutórios, indicações de leitura, recursos multimídia e atividades formativas (feedback automático). Ao final de cada módulo o estudante foi submetido a uma atividade avaliativa (objetiva), composta por dez questões de múltipla escolha, sendo o resultado desta a qualificação do aluno no módulo em questão. Cabe salientar que a estrutura descrita é padrão para os oito módulos do Algo+.

Com o objetivo de avaliar a experiência do aluno com o Portal Algo+, foram analisados dois conjuntos de informações coletadas durante o experimento: a observação das notas/médias dos discentes ao utilizar os módulos do Portal em comparação a seu desempenho em sala de aula e, através de um instrumento de pesquisa (questionário) respondido, pelos alunos, ao final das atividades do segundo módulo do Algo+.

A primeira análise dos resultados do experimento focou em um estudo quantitativo de desempenho dos alunos, nas avaliações realizadas no Algo + e, uma comparação em relação às notas obtidas em sala de aula (para esta observação adotou-se as notas da primeira avaliação da disciplina, visto que esta contemplou os mesmos assuntos abordados nos módulos utilizados do Algo + ). Para a análise estatística (SPSS ${ }^{1}$ ) das médias, inicialmente realizou-se um teste de normalidade (PARK, 2015), a fim de identificar o padrão de distribuição das notas observadas tanto em sala de aula, quanto na utilização dos módulos do Algo+. Assumiu-se uma hipótese $\mathrm{H} 0$, na qual os dados da variável originam-se de uma distribuição normal para um nível de significância $>0,05$. O resultado alcançado com o Teste de Shapiro-Wilk (ROYSTON, 1992) demonstrou um $p$-value de $0,000<0,05$, desta forma rejeitando H0 (Figura 05). Este resultado indicou a necessidade da utilização um método não paramétrico para a comparação das médias, o que levou ao emprego do teste de Wilcoxon (FIX \& HODGES JR, 1955).

Testes de Normalidade
\begin{tabular}{|l|r|r|r|r|r||r|}
\hline & \multicolumn{3}{|c|}{ Kolmogorov-Smirnov ${ }^{\text {a }}$} & \multicolumn{3}{|c|}{ Shapiro-Wilk } \\
\cline { 2 - 6 } & Estatistica & \multicolumn{1}{|c|}{ df } & \multicolumn{1}{c|}{ Sig. } & Estatística & \multicolumn{1}{c|}{ df } & Sig. \\
\hline SalaAula &, 196 & 47 &, 000 &, 800 & 47 &, 000 \\
AlgoPlus &, 232 & 47 &, 000 &, 819 & 47 &, 000 \\
\hline
\end{tabular}
a. Correlação de Significância de Lilliefors

Figura 05. Resultado do teste de normalidade

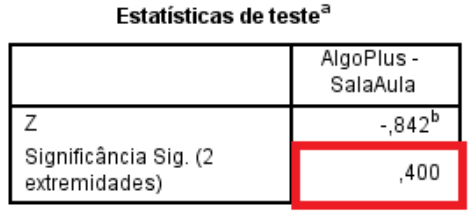

Figura 06. Resultado da comparação entre médias (Teste de Wilcoxon)

A Figura 06 demonstra o resultado da comparação das médias, o qual explicitou um grau de significância $0,400>0,05$, o que permitiu concluir que não existe diferença

\footnotetext{
1 Todos os testes estatísticos desta pesquisa foram realizados com o software IBM SPSS Modeler https://goo.gl/g7FXG7.
} 
estaticamente significativa entre as notas dos alunos ao realizaram provas em sala de aula e ao utilizarem os módulos do Portal. A evidência da proximidade das médias pode ser observada de forma mais clara no Gráfico 01.

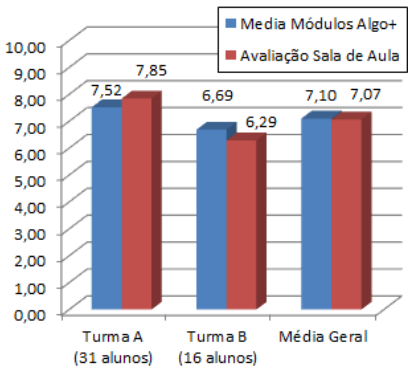

Gráfico 01. Comparativo entre notas/médias (Algo+ versus Sala de Aula)

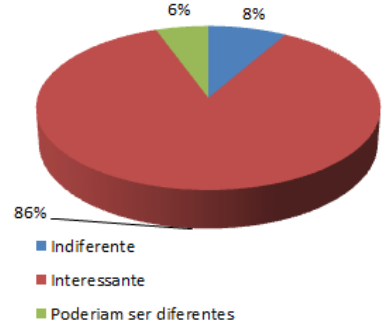

Gráfico 02. O que você achou da organização dos conteúdos em cada módulo do Portal?

A formalização do fato de que as médias dos alunos não se distinguem, neste ponto da pesquisa, pode ser considerado como um ponto positivo, visto que demonstrou que o conjunto de recursos disponibilizados no Portal, sua organização e o alinhamento a estratégias de ensino/aprendizagem permitiram ao discente realizar seus estudos de forma autônoma, sem a tutoria direta de um docente, possibilitando alcançar um desempenho não distinto ao obtido em sala de aula.

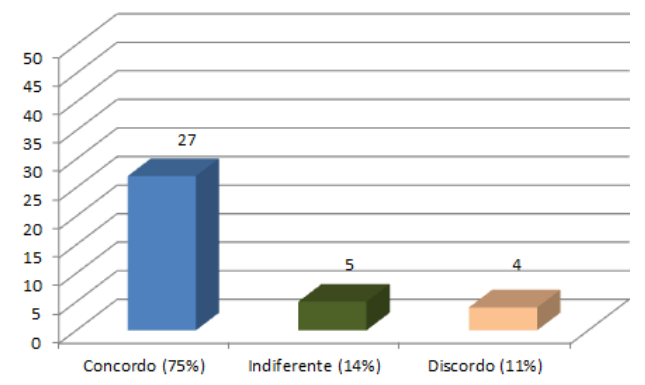

Gráfico 03. Você considera que a organização dos conteúdos de cada módulo estimulam o estudante a aprender sobre $o$ tema abordado?

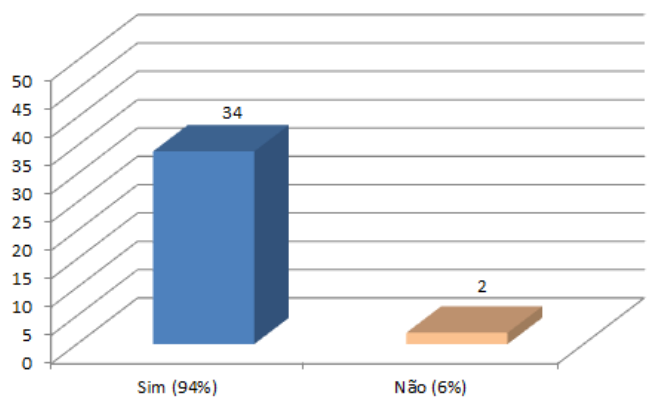

Gráfico 04. Você indicaria a utilização do Algo+ a outros colegas

Após a análise das notas, realizou-se a investigação do questionário respondido pelos estudantes. Este instrumento continha um conjunto de 27 questões (abertas e fechadas) e foi respondido por 36 discentes. Aqui serão apresentados os resultados de 4 perguntas, consideradas relevantes para este momento de discussão. Em relação a organização dos conteúdos disponibilizados no Portal (Gráfico 02), 86\% dos alunos (31) responderam que a ordem de conteúdos está interessante, 3 estudantes $(8 \%)$ apontaram indiferença sobre isto e $2(6 \%)$ sugeriram que poderia ser diferente. Em relação a características motivacionais foi questionado se a forma como os diferentes conteúdos estão disponibilizados no Algo+ estimulariam o aluno nas atividades de estudo (Gráfico 3 ), sendo que $75 \%$ dos alunos (27) concordaram, 14\% disseram ser indiferentes e $11 \%$ discordaram. Observa-se que os percentuais se aproximam nos gráficos 02 e 03 , o que corrobora para o entendimento que os materiais estão minimante alinhados com as necessidades de grande parte dos estudantes.

Foi questionado se os materiais/conteúdos dos módulos permitiram que o aluno, ao passar para o próximo módulo, tivessem subsídios suficientes do módulo anterior 
para o estudo dos novos conteúdos apresentados. Para esta questão 100\% dos estudantes acenaram positivamente, ou seja, as características de aprendizagem significativa inseridas no portal foram percebidas pelos discentes. Por fim, indagou-se se pela experiência de utilização do Portal, de seus recursos e organização, os alunos indicariam o mesmo para seus colegas, questão respondida de forma positiva por 34 dos 36 estudantes (Gráfico 04). Este fato demonstra que, além de alcançar os objetivos iniciais, este projeto possui um grande potencial de ser utilizado realmente como instrumento de apoio ao processo de aprendizagem de algoritmos.

\section{Conclusões}

Este extrato de pesquisa discorreu sobre o projeto e implementação de um Portal para servir como ferramenta de apoio ao processo de ensino e de aprendizagem de algoritmos e programação, denominado Algo+. A concepção desta solução, com o intuito de ser uma aplicação efetiva, foi desenvolvida sobre uma proposta de arquitetura baseada no alinhamento de todas as atividades do Portal com fundamentadas teorias de aprendizagem.

A utilização do Algo+ por um conjunto de estudantes possibilitou concluir que, efetivamente, sua organização permitiu que os discentes realizassem seus estudos de forma satisfatória. Um ponto interessante a ser descrito, refere-se ao fato da característica híbrida que a estrutura deste software concede, visto que propicia ao professor um recurso de acompanhamento de seus alunos, porém também pode ser utilizado como ferramenta autônoma para discente que desejem aprender programação.

\section{Referências}

AUSUBEL, D. P. Aquisição e retenção de conhecimentos: Uma perspectiva cognitiva, Lisboa: Editora Plátano, 2003.

Bervian, Pedro Alcino, Amado Luiz Cervo, e Roberto da SILVA. "Metodologia científica." São Paulo: Pretence Hall (2002): 482-493.

BORGES, Marcos AF. Avaliação de uma metodologia alternativa para a aprendizagem de programação. In: VIII Workshop de Educação em Computação-WEI. 2000.

DETERS, Janice Inês, et al. "O desafio de trabalhar com alunos repetentes na disciplina de Algoritmos e Programação." Simpósio Brasileiro de Informática na Educação (2008).

DO CANTO FILHO, A. B., MÜLlER, T. J., AMARAL, E.M.H., DE LIMA, J. V., e Tarouco, L. M. R. Um Sistema para Classificação de Objetos de Aprendizagem MCS-Multimodality Classification System. RENOTE, 11(3), 2013.

AMARAL, Érico Marcelo Hoff do. "Processo de ensino e aprendizagem de algoritmos integrando ambientes imersivos e o paradigma de blocos de programação visual." Tese de Doutorado, PPGIE/UFRGS, 2015.

FARACO, R.A.; ROSATELLI, M.C., A learning companion system for distance education in computer Science. Anais do XII WEI, Salvador, SBC, 2004.

FINCHER, S.; COOPER, S.; KÖLlING, M.; MALONEY, J. Comparing Alice, Greenfoot \& Scratch. Proceedings do 41st SIGCSE'10, Wiscosin, USA, 2010. 
VI Congresso Brasileiro de Informática na Educação (CBIE 2017)

Anais do XXVIII Simpósio Brasileiro de Informática na Educação (SBIE 2017)

FIX, Evelyn, and J. L. Hodges Jr. "Significance probabilities of the Wilcoxon test." The Annals of Mathematical Statistics (1955): 301-312.

GOMES, Anabela et al. Aprendizagem de programação de computadores: dificuldades e ferramentas de suporte. Revista Portuguesa de Pedagogia, n. 42-2, p. p. 161-179, 2008.

KOLB, David A. Experiential learning: Experience as the source of learning and development. FT press, 2014.

KUHN, Thomas. A estrutura das revoluções científicas. 5. ed. São Paulo: Perspectiva, (Coleção Debates), 1998.

LEHFELD, Neide Aparecida de Souza, and A. de JP Barros. "Projeto de pesquisa: propostas metodológicas." Petrópolis: Vozes (1991).

PARK, Hun Myoung. "Univariate analysis and normality test using SAS, Stata, and SPSS." (2015).

RESNICK, M.; MALONEY,- J.; MONROY-HERNÁNDEZ, A.; RUSK, N.; EASTMOND, E.; BRENNAN, K.; MILLNER, A.; ROSENBAUM, E.; PRATA, J.; SILVERMAN, B.; KAFAI, Y. Magazine Communications of the ACM - Scratch Programming for All CACM, Volume 52, Páginas 60-67, 2009.

ROCHA, H. V. Representações Computacionais Auxiliares de Conceitos de Programação. In: "Computadores e Conhecimento: Repensando a Educação". Livro organizado por Valente, J. A. Editora Unicamp, 1993.

ROZAS, M. Rosa et al. Autoevaluación del aprendizaje en enfermería con ejercicios interactivos del programa Hot Potatoes. Educación médica, v. 11, n. 1, p. 19-27, 2008 .

ROYSTON, Patrick. "Approximating the Shapiro-Wilk W-Test for nonnormality." Statistics and Computing 2.3 (1992): 117-119.

SAIKKONEN, Riku; MALMI, Lauri; KORHONEN, Ari. "Fully Automatic Assessment of Programming Exercises". Annual Joint Conference Integrating Technology into Computer Science Education. Canterbury, Reino Unido. 2001.

SILVA, I. F. A.; SILVA, I. M. M.; SANTOS, M. S. Análise de problemas e soluções aplicadas ao ensino de disciplinas introdutórias de programação. Universidade Federal Rural de Pernambuco, Recife - PE. 03 pp.

VIZCAINO, A; CONTRERAS, J.; FAVELA, J.; PRIETO, M., Collaborative Environment to Develop Good Habits in Programming. Intelligent Tutoring Systems, Montreal, 2000.

WANG, Marco Alberto; PRADO, Edmir Parada Vasques. Revisão Sistemática sobre Alfabetização Computacional. XI Simpósio Brasileiro de Sistemas de Informação (SBSI), Goiânia/GO, 2015. 